\title{
Discurso inspirado en Bateson. El Patrimonio, el Físico Ambiental y el Restaurador
}

Marco Pretelli, Kristian Fabbri, Leila Signorelli | Università degli Studi di Bologna, Dip. Architettura

URL de la contribución <www.iaph.es/revistaph/index.php/revistaph/article/view/3662>

El Patrimonio (EP): ¿Estoy permaneciendo al margen de la cuestión de la eficiencia energética?

El Físico Ambiental (FA): Sí, y igracias a Dios! Esperamos que siga así aún mucho tiempo...

El Restaurador ( $R$ ): ¿Qué dices? Yo tendría que decir "gracias a Dios".

FA: Es que tú $(R)$ piensas que las nuestras son posicio- nes opuestas, pero no es cierto.

R: Querido (FA), por lo general los que estudian la eficiencia energética piensan en ella como "pura", sin hacer un estudio histórico del edificio para saber lo que va a trabajar.

FA: Es una cuestión de perspectiva. Tradicionalmente el conocimiento técnico se ha desarrollado olvidando la

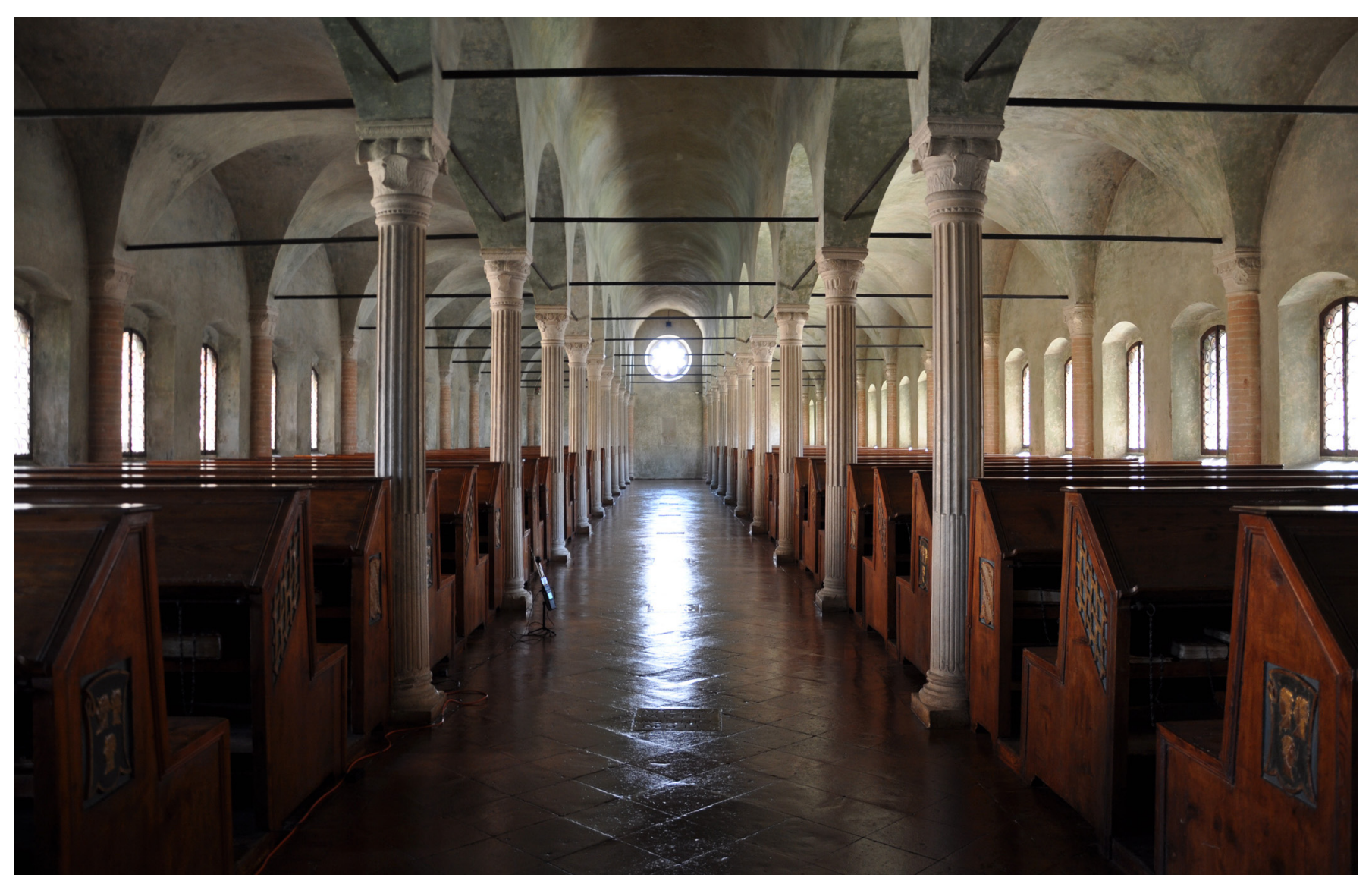

Biblioteca Malatestiana en Cesena (FC), la única parte conservada de un antiguo edificio que ha sobrevivido sin instalaciones (ni calefacción, ni iluminación) | foto Kristian Fabbri 
a debate La eficiencia energética y la edificación histórica | coordinan Mónica López Sánchez, Ana Yáñez Vega

historia... es difícil encontrar apasionados del Motorola Startack, hoy siempre se elige un Smartphone. Pero también está la historia de la tecnología en el sector de la construcción, que lo restauradores han ignorado (ignorantes).

R: Así que el conocimiento del edificio histórico es la forma de encontrar soluciones que mejoren la respuesta energética del edificio sin alterarlo. ¿Estás de acuerdo? FA: ¡Exactamente! Investigación de archivo, monitoreo, modelación $3 d$... Todas herramientas esenciales.

EP: Interesante. Si la respuesta está dentro de mí no estoy tan "al margen" de la cuestión.

\section{Entonces: la Eficiencia Energética c'est moi!}

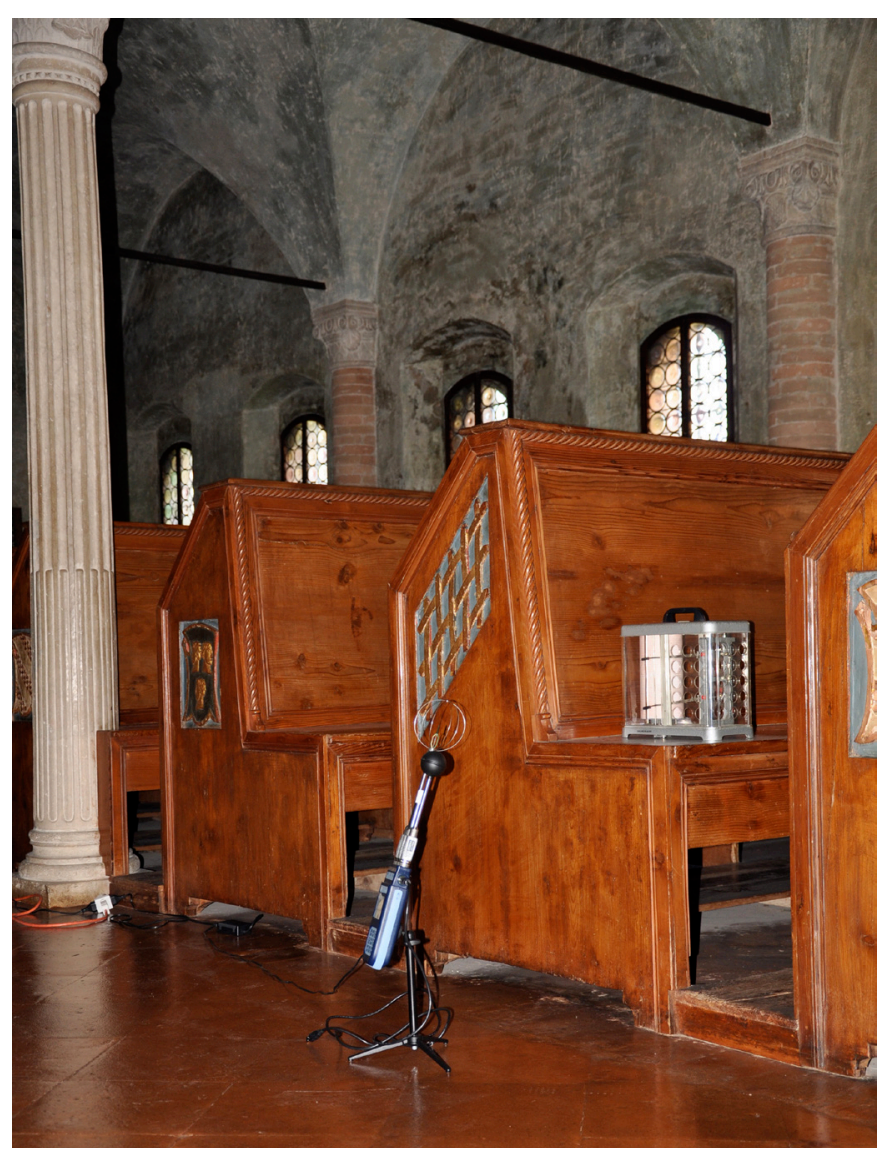

Detalle de la sonda para el monitoreo del microclima, instalada en la biblioteca | foto Kristian Fabbri

\section{BIBLIOGRAFÍA}

- FABBRI, K.; PRETELLI, M. (2014) Heritage buildings and historic microclimate without HVAC technology: Malatestiana Library in Cesena, Italy, UNESCO Memory of the World. Energy \& Buildings, 76, 2014, pp. 15-31

- PRETELLI, M.; SIGNORELLI, L. (2014) Sustainability between economy and culture. Restoration, re-construction and "reuse" in some post-world war II cases in Germany (East and West). En BISCONTIN, G.; DRIUSSI, G. (a cura di) Quale sostenibilita' per il restauro. Atti del convegno internazionale (Bressanone 1-14 luglio 2014). Marghera-Venezia: Edizioni Arcadia Ricerche srl, 2014, pp. 41-51

- PRETELLI, M.; UGOLINI, A.; FABBRI, K. et ál. (2013) Indoor Microclimate effect on heritage buildings: the case study of Malatestiana Library. En BORIANI, M.; GABAGLIO, R.; GULGOTTA, D. (a cura di) BUILT HERITAGE 2013. Monitoring Conservation and Management. Attidel convegno internazionale (Milano 18-20 novembre 2013). Milano: Politecnico di Milano, Centro per la Conservazione e Valorizzazione, 2013, pp.14391446

- PRETELLI, M.; UGOLINI, A.; FABBRI, K. (2013) "Historic plants as monuments" preserving, rethinking and re-using historic plants. Journal of Cultural Heritage, vol. 14, n. ${ }^{\circ} 3,2013$, pp. S38-S43

- PRETELLI, M.; UGOLINI, A.; FABBRI, K. (2012) "Historic plants as monuments" preserving, rethinking and re-using historic plants. En PROCEEDINGS 5th International Congress on "Science and Technology for the Safeguard of Cultural Heritage in the Mediterranean Basin" Vol. II, Diagnostics and restoration (1st Part) (Reprint) (Istanbul, Turkey 22-25 November 2011). Roma: Valmar, 2012, pp. 18-23

- UGOLINI, A.; FABBRI, K.; MEI, C. et ál. (2014) SUSTAINIBILITY as "I CARE" Maintenance and sustainability for the Italian-Swiss Centre of Rimini. En BISCONTIN, G.; DRIUSSI, G. (a cura di) Quale sostenibilita' per il restauro. Atti del convegno internazionale (Bressanone 1-14 luglio 2014). Marghera-Venezia: Edizioni Arcadia Ricerche srl, 2014, pp. 189-199 